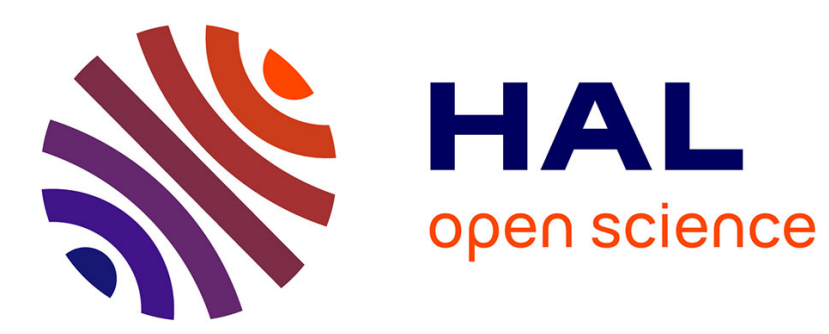

\title{
Phase evaluation for electronic speckle-pattern interferometry deformation analyses
}

Sanichiro Yoshida, Dr. Suprapedi, Rini Widiastuti, Edi Tri Astuti, Anung Kusnowo

\section{- To cite this version:}

Sanichiro Yoshida, Dr. Suprapedi, Rini Widiastuti, Edi Tri Astuti, Anung Kusnowo. Phase evaluation for electronic speckle-pattern interferometry deformation analyses. Applied optics, 1995, 20 (7), pp.755-757. hal-01092292

\section{HAL Id: hal-01092292 \\ https://hal.science/hal-01092292}

Submitted on 8 Dec 2014

HAL is a multi-disciplinary open access archive for the deposit and dissemination of scientific research documents, whether they are published or not. The documents may come from teaching and research institutions in France or abroad, or from public or private research centers.
L'archive ouverte pluridisciplinaire HAL, est destinée au dépôt et à la diffusion de documents scientifiques de niveau recherche, publiés ou non, émanant des établissements d'enseignement et de recherche français ou étrangers, des laboratoires publics ou privés. 


\title{
Phase evaluation for electronic speckle-pattern interferometry deformation analyses
}

\author{
Sanichiro Yoshida, Suprapedi, Rini Widiastuti, Edi Tri Astuti, and Anung Kusnowo \\ Research and Development Center for Applied Physirs, Indonesiun Institute of Sciences, \\ Puspiptek, Serpong, Tangerang 15310, Indonesia
}

\begin{abstract}
A new, handy method to evaluate phase data from fringe patterns produced by electronic speckle-pattern interferometry is proposed. The method is capable of evaluating phase data by simply taking speckle images and performing arithmetic operations on them. No extra optics or phase modulation is needed. Experiments have been carried out to prove the validity of the principle and demonstrate the capability of applications to practical deformation analyses.
\end{abstract}

Electronic speckle-pattern interferometry (ESPI) is now widely used for analyses of various types of deformation and displacement. ${ }^{1}$ The advantage of ESPI is that, unlike conventional photographic methods, ${ }^{2.3}$ it enables one to evaluate the phase data embedded in a fringe pattern continuously rather than discretely at the locations of fringes (dark peaks). A number of techniques to evaluate phase data have been demonstrated. These techniques can be classified into two types, i.e., the Fourier-transform type, ${ }^{4}$ which makes use of spatial carrier fringes, and the phase-stepping type, ${ }^{5}$ which makes use of temporal carriers. There are many reports in which these techniques are successfully applied to practical applications. ${ }^{6}$ However, both types of technique essentially require the introduction of carrier fringes, and thereby an optical rearrangement such as wave-front transformation or phase shifting between exposures is inevitable. It is obviously preferable to use a phase-evaluation method in which such optical rearrangement is not necessary.

In this Letter we propose a new method to evaluate phase data and demonstrate the validity of the proposed method by applying it to in-plane displacement measurement. The advantages of the new method are that (i) optical rearrangement between exposures is not needed and (ii) computation time is extremely short because no mathematical procedure other than simple arithmetic operations is needed. Advantage (i) leads to the additional advantage that the system is free from the error caused by optical rearrangement.

Figure 1 illustrates the optical setup of our scheme. The interferometric arrangement is basically the same as for conventional dual-beam ESPI, ${ }^{2}$ except that there is a mechanism to block the two interferometric branches one by one. The propagation vectors of the two interferometric branches lie in a plane parallel to the optical table $(z-x$ plane $)$ and cross each other on the target plane ( $x-y$ plane) with a common incident angle. Thus this interferometer is sensitive to the $x$ component of in-plane displacement of the object. The CCD camera used is made of a two-dimensional array of $510 \times 510$ pixels, each of which has a detection area of $8.4 \mu \mathrm{m} \times 9.8 \mu \mathrm{m}$ (horizontal/vertical).

In this method the phase data are evaluated in three steps. In the first step (image acquisition step), speckle images of the object with illumination by the first interferometric branch (singleillumination image $I_{1}$ ), by the second interferometric branch (single-illumination image $I_{2}$ ), and by both branches simultaneously (dual-illumination image) are taken by the CCD camera and stored in a frame memory. The background noise is also stored. Then, after the object undergoes the displacement to be measured, another dual-illumination image is taken. The dual-illumination images taken before and after the displacement can be written as

$$
\begin{aligned}
I_{\text {before }} & =\mid I_{0}[1+\gamma \cos (\theta)]+\text { noise } \mid, \\
I_{\text {after }} & =\mid I_{0}[1+\gamma \cos (\theta+\phi)]+\text { noise } \mid,
\end{aligned}
$$

where $I_{0}=I_{1}+I_{2}$, where $I_{1}$ and $I_{2}$ are the intensities of the first and the second interferometric branches, respectively, $\theta$ is the initial phase difference between the two interferometric branches, $\gamma$ is the visibility, and $\phi$ is the change in phase difference caused by the displacement.

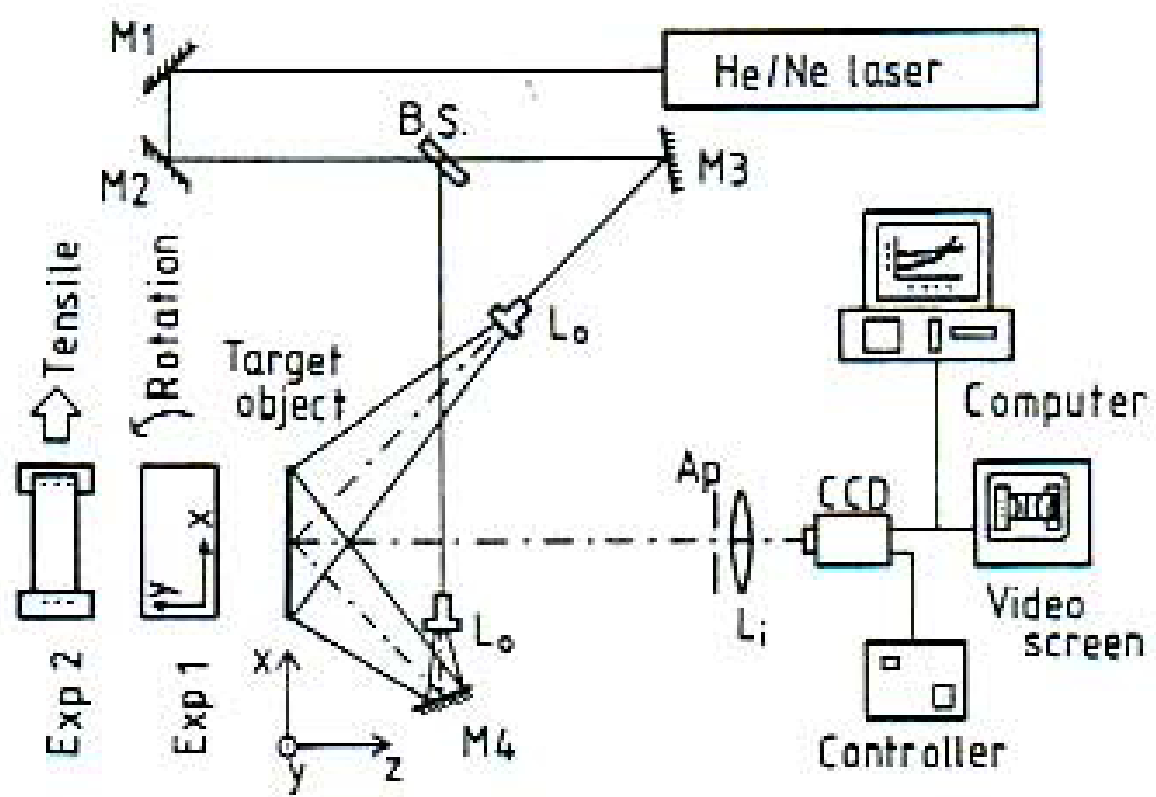

Fig. 1. Experimental setup. Two types of target object were investigated. M1-M4, mirrors; B.S., beam splitter; Ap, aperture; $L_{0}$ 's, objective lenses; $L_{i}$, imaging lens. 
In the second step (image processing step), the background noise is subtracted from all the images taken in the image acquisition step. Then addition and subtraction of $I_{\mathrm{befure}}$ and $I_{\text {after }}$ are performed. The result of the addition is further modified by subtraction of $2\left(I_{1}+I_{2}\right)$ so the de term $2 I_{0}$ can be eliminated. This step results in two modified fringe systems, which can be written as

$$
\begin{aligned}
& I_{\text {addmad }}=\left|2 I_{0} \gamma \cos (\theta+\phi / 2) \cos (\phi / 2)\right|, \\
& I_{\text {submod }}=\left|2 I_{0} \gamma \sin (\theta+\phi / 2) \sin (\phi / 2)\right| .
\end{aligned}
$$

In the third step (numerical operation step), the intensity data contained in the pixels along a line perpendicular to the fringe are averaged over a certain number of adjacent pixel lines. The averaging is done over the same pixel lines for the $I_{\text {sddmod }}$ and $I_{\text {sabmod }}$ images. As the phase value $\phi$ is constant along the direction parallel to the fringe, this averaging can be expressed as

$$
\begin{aligned}
\left\langle I_{\text {addmod }}\right\rangle & =\left(2 I_{0} \gamma / N\right)|\cos (\phi / 2)| \sum_{i}^{N}\left|\cos \left(\theta_{i}+\phi / 2\right)\right|, \\
\left\langle I_{\text {eubmod }}\right\rangle & =\left(2 I_{0} \gamma / N\right)|\sin (\phi / 2)| \sum_{i}^{N}\left|\sin \left(\theta_{i}+\phi / 2\right)\right|,
\end{aligned}
$$

where $N$ is the number of the pixels involved in the averaging. Because the random phases $\theta_{i}$ are uniformly distributed over all values between 0 and $2 \pi$, the values of $\sum_{i}^{N}\left|\cos \left(\theta_{i}+\phi / 2\right)\right|$ and $\sum_{i}^{N} \mid \sin \left(\theta_{i}+\right.$ $\phi / 2) \mid$ approach each other as $N$ is increased. Therefore, if $N$ is sufficiently large, the phase $\phi / 2$ can be evaluated as

$$
\phi / 2=\tan ^{-1}\left(\left\langle I_{\text {submod }}\right\rangle /\left\langle I_{\text {sdd mad }}\right\rangle\right) .
$$

We examined this method for two types of in-plane displacement. In the first experiment we gave a small rotation to a target object (aluminum plate) about an axis normal to the object plane ( $z$ axis). Such a movement causes a displacement in which the $x$ component $u$ is written as $u=\delta y$, where $\delta$ is the rotation angle, and thereby produces an equidistant fringe system parallel to the $x$ axis. In the second experiment we gave a tensile deformation to an aluminum object, which resulted in a nonparallel fringe system. In both experiments the phase change $\phi / 2$ is related to displacement $u$ by $\phi / 2=$ $(2 \pi / \lambda) u \sin \alpha=(\sqrt{2} \pi / \lambda) u$, where $\alpha$ is the angle of incidence $\left(45^{\circ}\right)$.

Figure 2 shows video-screen printouts displaying $I_{\text {addmod }}$ and $I_{\text {sabmod }}$ fringe patterns obtained when the target plate was rotated by $\sim 0.03$ mrad. The screw (the larger one) imaged at the lower left-hand corner of the print-out is the center of rotation, and a line drawn through the screw represents the $x$ axis. Both images represent equidistant, parallel fringe systems. Knowing that the $x$ component of displacement is zero on the $x$ axis, one can determine the absolute value of $\phi / 2$ for each fringe, as shown in the middle of Fig. 2. It is clearly seen that the fringes of $I_{\text {subrod }}$ are located at $\phi / 2=\ldots, 0, \pi, 2 \pi, \ldots$, and those of $I_{\text {addmod }}$ are at $\phi / 2=\ldots,-\pi / 2,+\pi / 2, \ldots$.
This proves that both images properly represent the sine and cosine characteristics caused by the terms containing $\phi / 2$ alone in the arguments in Eqs. (3) and (4), respectively.

In the lower part of Fig. 2 the intensity profiles obtained by averaging ten pixel lines around a representative line $x=250$ are shown. We read the vertical coordinate $y(\mathrm{~mm})$ from the video print-out, using the ruler imaged at the right-hand side. The profiles at the left and at the right represent the sine and cosine profiles, respectively. In the $I_{\text {addmod }}$ profile a comparison is made among the results obtained by averaging ten pixel lines (case 3) and five pixel lines (case 2) and no averaging (case 1). As the number of averaged lines increases, the high-frequency fluctuation caused by $\langle\cos (\theta+\phi / 2)\rangle$ diminishes, and consequently the profile represents the term $\cos (\phi / 2)$ more clearly.

With the use of Eq. (7) we evaluated phase data along the representative lines shown in Fig. 2. The results are shown in Fig. 3 for cases 1-3. For clarity, data for case 1 and case 2 are shifted vertically. The solid lines represent the theoretical linearity between $\phi / 2$ and $y ; \phi / 2=(\sqrt{2} \pi / \lambda) u=$ $(\sqrt{2} \pi / \lambda) \delta y$. We drew these lines in such a way
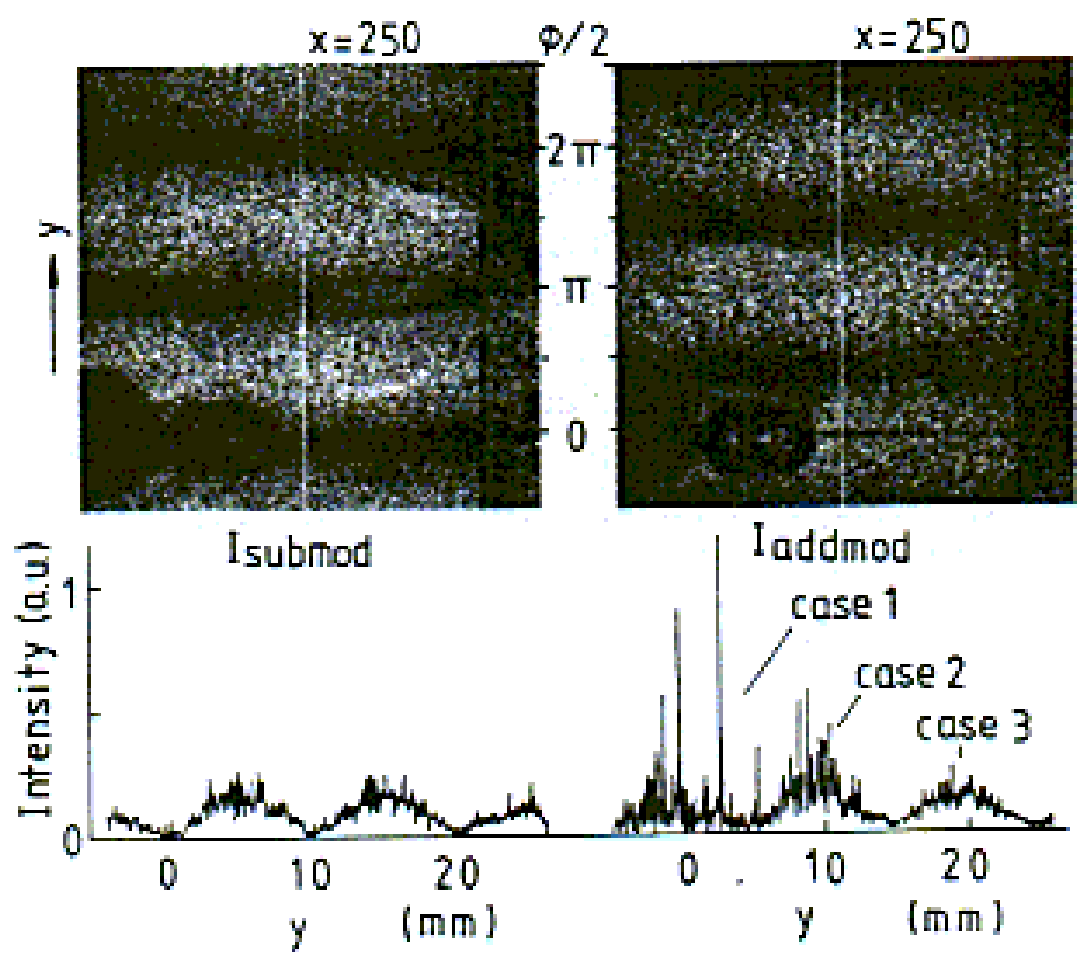

Fig. 2. $I_{\text {submod }}$ and $I_{\text {addmod }}$ fringe patterns and intensity profiles at a representative line. The three periods of $I_{\text {addmod }}$ compare different cases of averaging.

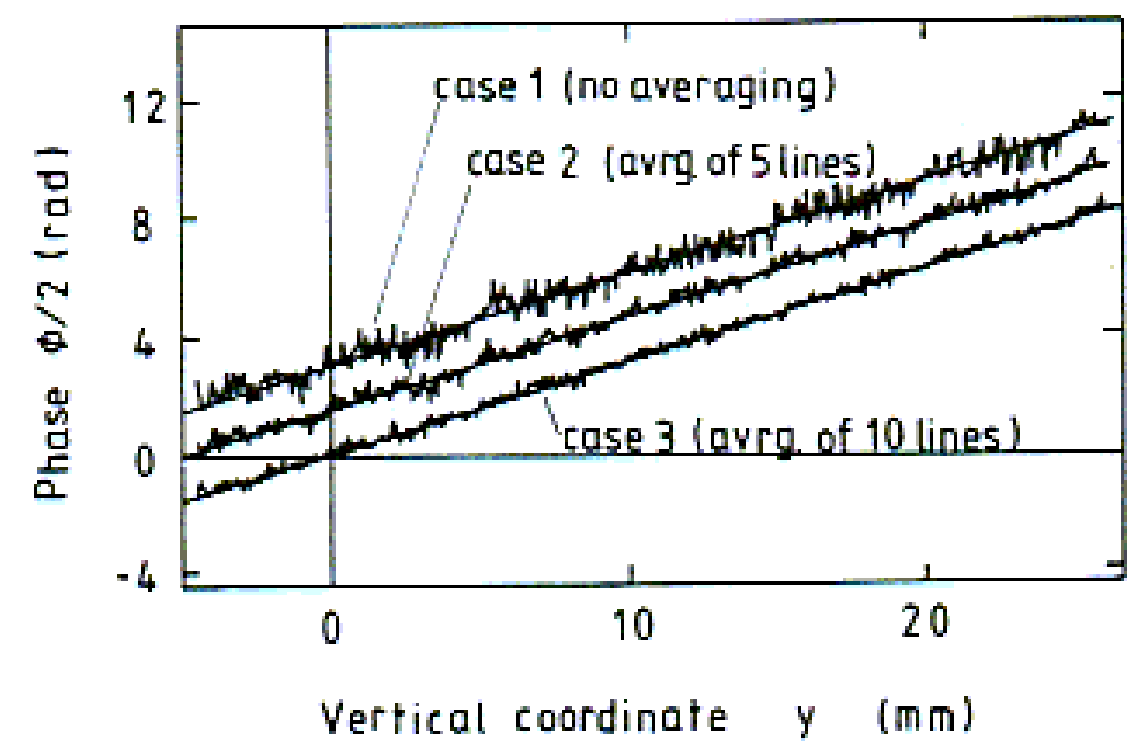

Fig. 3. Phase data evaluated in experiment 1. For clarity, curves for case 1 and case 2 are shifted vertically. 


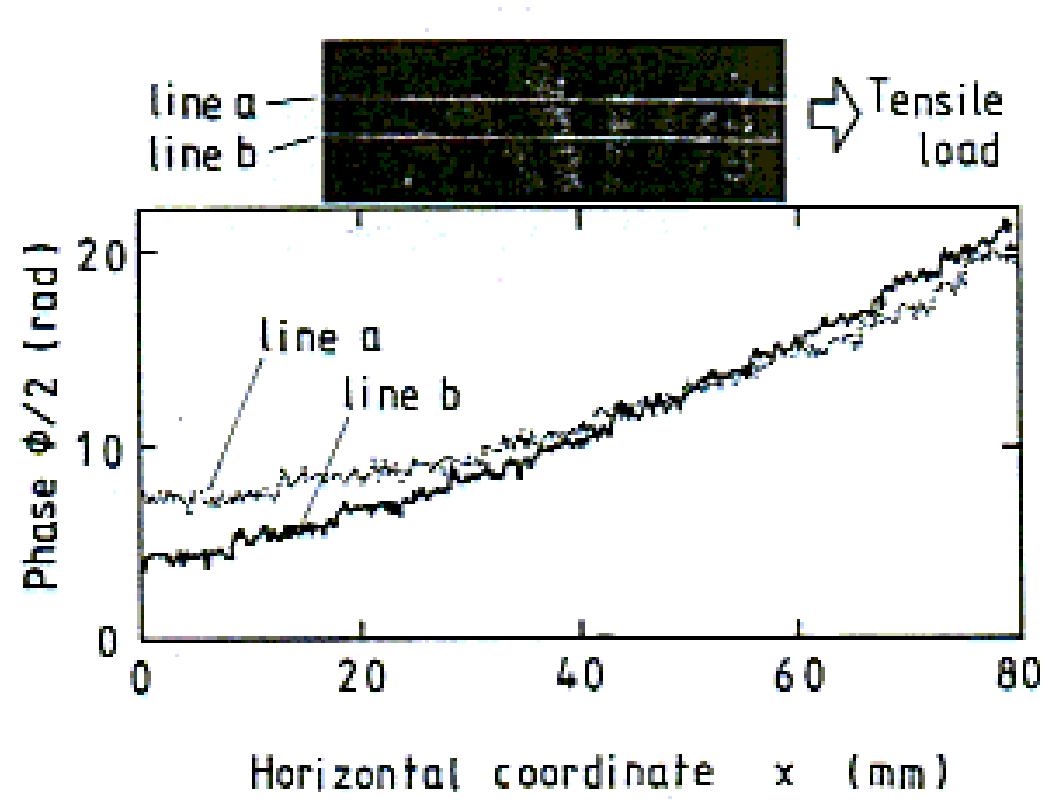

Fig. 4. Phase data evaluated in experiment 2.

that they could pass through two reference points $(y, \phi / 2)=(0,0),(20,2 \pi)$, where the values of $y$ were determined on the $I_{\text {submod }}$ pattern in Fig. 2. We did not use $\delta$ to draw a theoretical line because the measurement of such a small angle was inexact.

Thus a comparison of evaluated data with the theoretical lines measures the accuracy of our method in terms of (i) the linearity between $\phi / 2$ and $y$ and (ii) the degree of high-frequency fluctuation caused by 0 . Both factors are associated with the accuracy of approximation $\sum_{i}^{N}\left|\cos \left(\theta_{i}+\phi / 2\right)\right|=\sum_{i}^{N}\left|\sin \left(\theta_{i}+\phi / 2\right)\right|$ that we made to derive Eq. (7). In all cases presented in Fig. 3, clear linearity is observed. When ten pixel lines were averaged (case 3 ), the deviation of the evaluated phase data from the theoretical lines (called the phase-determination error) is $\pm 0.3 \mathrm{rad}$. The phase-determination errors for the other cases are $\pm 0.6 \mathrm{rad}$ (case 2 ) and $\pm 1 \mathrm{rad}$ (case 1 ), respectively.

Next we applied the present method to a practical deformation analysis. We applied a tensile load to an aluminum plate, which caused the nonparallel fringe system shown in Fig. 4. We evaluated the phase as a function of the horizontal coordinate $x$ for two representative lines, lines a and $b$. To decrease the phase-determination error requires that the pixel data be averaged over some parallel pixel lines adjacent to the representative line. As the fringes are not parallel, such pixel lines do not cross some of the fringes perpendicularly. In those regions an error occurs in evaluation of $\phi$ because $\phi$ does not keep exactly the same value during the averaging. Therefore it is preferable to have the number of pixel lines being averaged small. We selected this number to be three.

The evaluated phase data are shown in Fig. 4. The resultant phase-deformation error was $\pm 0.7 \mathrm{rad}$. The width of lines $a$ and $b$ shows the width of three pixel lines. Note that this width is satisfactorily small in comparison with the spatial variation of $\phi$. The error caused by averaging pixels over these lines in a direction not parallel to the fringe at the left-hand end of the image is $\pm 0.1 \mathrm{rad}$. This value is somewhat smaller than the phasedetermination error.
Finally, we discuss the conditions for which the new method is indicated. Because Eqs. (1)-(7) are valid for the dual-beam ESPI universally, our method is basically applicable to other dual-beam ESPI setups, provided that the conventional subtraction fringe pattern is obtainable and that the average intensity $I_{0}$ is measurable. As is the case for phasestepping methods, ${ }^{5}$ however, the accuracy of the phase evaluation is dependent on the visibility $\gamma$, which appears in Eq. (1) and can be expressed in general as $\left[2 \sqrt{\left(I_{1} I_{2}\right)} / I_{0}\right] \gamma_{0}$, where $\gamma_{0}$ is the amplitude of the complex degree of mutual coherence. Thus, using the rotating target (Exp. 1 in Fig. 1), we made an additional series of experiments in which we evaluated the phase-determination error for various $\gamma$. We varied $\gamma$ in two ways, i.e., by changing the optical path difference between the two interferometric branches (causing a change in $\gamma_{0}$ ) and by changing the intensity ratio $I_{1} / I_{2}$ (causing a change in $\left.2 \sqrt{\left(I_{1} I_{2}\right)} / I_{0}\right)$. We evaluated the resultant $\gamma$ as $\left(I_{\max }-I_{\min }\right) /\left(I_{\max }+I_{\min }\right)$, where $I_{\max }$ and $I_{\min }$ are, respectively, the intensities of the brightest and darkest peaks of the fringe pattern. Note that the $\gamma$ dependence of the phase-determination error discussed below was irrelcvant to the choice of the way to vary $\gamma$ and that the $I_{\text {gddmod }}$ and $I_{\text {submod }}$ obtained from the same pair of $I_{\text {before }}$ and $I_{\text {after }}$ showed the same $\gamma$, within an error of $\sim 10 \%$. When $\gamma$ was progressively reduced from 0.9 to 0.4 , first the phase-determination error increased slowly until $\gamma$ became $\sim 0.6$, and then the rate of increase grew considerably. The phasedetermination errors observed when $\gamma=0.7,0.6,0.5$, 0.4 were larger than that at $\gamma=0.9$ by $\sim 5 \%, \sim 10 \%$, $\sim 25 \%$, and $\sim 50 \%$, respectively.

In conclusion, a new phase-evaluation method has been proved to be valid and useful. We believe that this method is extremely powerful for ESPI deformation analyses in which the object undergoes dynamic and random deformation and thereby it is essential to measure the deformation successively with a given, presumably small, time interval.

The authors are grateful to Satoru Toyooka of Saitama University for helpful discussions. The assistance of Syahroni, Tetty M. Purba, and Rita Juliani is highly appreciated. This research is supported by the Research and Development Corporation of Japan.

\section{References}

1. G. Gulker, K. Hinsch, C. Holscher, and H. Neunaber, Opt. Eng. 29, 816 (1990).

2. J. A. Leendertz, J. Phys, E 3, 214 (1970).

3. E. Archbold, J. M. Burch, and A. E. Ennos, Opt. Acta 17, $883(1970)$.

4. D. Kerr, F. Mendoza Santoyo, and J. R. Tyrer, J. Mod. Opt. 36, 195 (1989).

5. D. Kerr, F. Mendoza Santoyo, and J. R. Tyrer, J. Opt. Soc. Am. A 7, 820 (1990).

6. F. Mendoza Santoyo, M. C. Shellabear, and J. R. Tyrer, Appl. Opt. 30, 717 (1991). 